\title{
SKRJABINELAZIA GALLIARDI (NEMATODA, SEURATOIDEA) : COMPLÉMENTS MORPHOLOGIQUES ET CYCLE BIOLOGIQUE
}

\author{
A. G. CHABAUD*, O. BAIN*, G. O. POINAR, Jr.**
}

RÉSUMÉ. Skrjabinelazia galliardi n'était connu que par la morphologie des femelles. Un nouveau matériel permet de compléter l'étude morphologique et de mieux comprendre la biologie.

Le mâle, la larve du $3^{\text {e }}$ stade et la larve du $4^{\text {e }}$ stade sont décrits.

Les jeunes femelles pondent un très petit nombre de larves du $3^{e}$ stade, ce qui permet une faible reproduction de type Atractide, c'est-à-dire une multiplication des parasites directement à l'intéricur de l'hòte. Les vieilles femelles sont ovipares, mais les œufs contiennent une larve

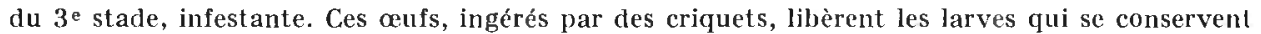
telles qu'elles chez l'insecte. Le lézard entomophage, hôte définitif, qui n'a pas dans la nature l'opportunité d'ingérer des oufs, peut donc être infesté par ingestion d'insectes.

Le cycle biologique est donc intermédiaire entre le type Cosmocercide, avec développement des larves dans le milieu extérieur et le type Spiruride avec développenent des larves chez un arthropode.

Mots-clés : Skrjabinelazia. Seuratoidea. Gonadotes. Biologie de Skrjabinelazia.

\section{Skrjabinelazia galliardi (Nematoda, Seuratoidea): further morphological data and life history.}

SUMMARY. Skrjabinelazia galliardi was previously known only from a description of the morphological characteristies of the female. Additional mal erial being available, we were able to complete the morphological description and understand better the biology of the species.

The male, the third stage larva and the fourth stage larva are described.

Young females lay a very small number of third stage larvae, leadling in a small scale to a reproduction of the atracticle-type, that is a multiplication of the parasite directly insicle the host. The old females are oviparous, but eggs contain a third stage infective larva. These eggs, after being ingested by field-crickets, hatch and without further development remain as larvae in the insect. The definitive entomophagous lizard host, which, in the field, has no opportunity to

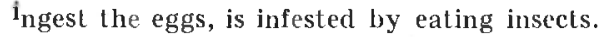

So, the life history is intermediary between the cosmocercid like cycle (with three larval stages) and the spirurid-like cycle (with development of larval stages in an invertebrate).

Key-words: Skrjabinelazia. Seuratoidea. Gonadotes. Biology of Skrjabinelazia.

* Laboratoire de Zoologie (Vers), associé au CNRS, Muséum National d'Hisloire Naturelle, 61, rue Bu/lon, $F 75231$ Paris Cedex 0.3.

** Entomology and Parasitology, Agricullural Experiment Station, Lniversity of California, Berkeley, 201 Wellman Hall, Berkeley, California 94720, USA.

Accepté le 29 février 1988. 
Les nématodes phasmidiens, parasites de vertébrés, dérivent tous d'ancêtres proches des Rhabditides actuels, et paraissent monophylétiques. Ils se sont diversifiés en plusieurs grands groupes, très riches en espèces, relativement homogènes par la morphologie aussi bien que par la biologie. En outre, quelques genres mal connus, souvent de petite taille, pauvres en espèces, font la transition entre ces grands groupes. Leur étude est particulièrement importante pour tenter de comprendre comment a pu s'effectuer l'évolution des grands ensembles et le passage de l'un à l'autre.

Skrjabinelazia galliardi Chabaud, 1973, parasite d'un lézard Sphaerodactylidae brésilien Gonadotes humeralis Guichenot, 1855 est un bon exemple de ces espèces de transition.

La femelle a été décrite dans un premier temps mais le mâle et le cycle biologique sont restés inconnus.

De nouveaux lézards, récoltés dans les mêmes gites que précédemment ont pu être examinés. La plupart étaient négatifs, mais l'un d'eux était bien positif avec deux mâles, deux femelles à œufs rouges et une larve du $4^{\mathrm{e}}$ stade dans l'estomac; deux femelles à œufs rouges et quatre femelles avec de rares oufs blancs (1 à 12) embryonnés dont une avec une larve libre dans l'intestin.

Il a donc été possible de compléter les données morphologiques par la description du mâle, de la larve infestante au $3^{\mathrm{e}}$ stade et de la larve au $4^{\mathrm{e}}$ stade. En outre, les femelles mùres ont été utilisées pour infester expérimentalement des invertébrés et élucider le cycle biologique.

\section{I - Compléments morphologiques}

A - Mal.e

Corps long de $1600 \mu \mathrm{m}$, enroulé en crosse dans la région postérieure ; largeur maximum $50 \mu \mathrm{m}$; tête avec plateau cuticulaire épais ; anneau nerveux à $130 \mu \mathrm{m}$ de l'apex ; cesophage long de $230 \mu \mathrm{m}$; musculature précloacale puissante ; spicule long de $40 \mu \mathrm{m}$, à extrémité distale pointue ; orifice cloacal sur une protubérance ; 3 paires de papilles : une au niveau du cloaque et deux sur la queue; extrémité caudale arrondie et ornée de petites pointes.

LARVE INFESTANTE DU TROISIÈME STADE

Une larve extraite d'un ouf rouge est longue de $400 \mu \mathrm{m}$ et large de $21 \mu \mathrm{m}$; la tête est terminée par une dent cuticulaire latérale, obtuse ; à la base sont visibles 4 papilles ; pore excréteur et anneau nerveux à 85 et $120 \mu \mathrm{m}$ de l'apex; œsophage rhabditoïde long de $170 \mu \mathrm{m}$; ébauche génitale à $210 \mu \mathrm{m}$ de l'apex; queue longue de $55 \mu \mathrm{m}$, terminée par une pointe dont la base est soulignée par un sillon cuticulaire. 


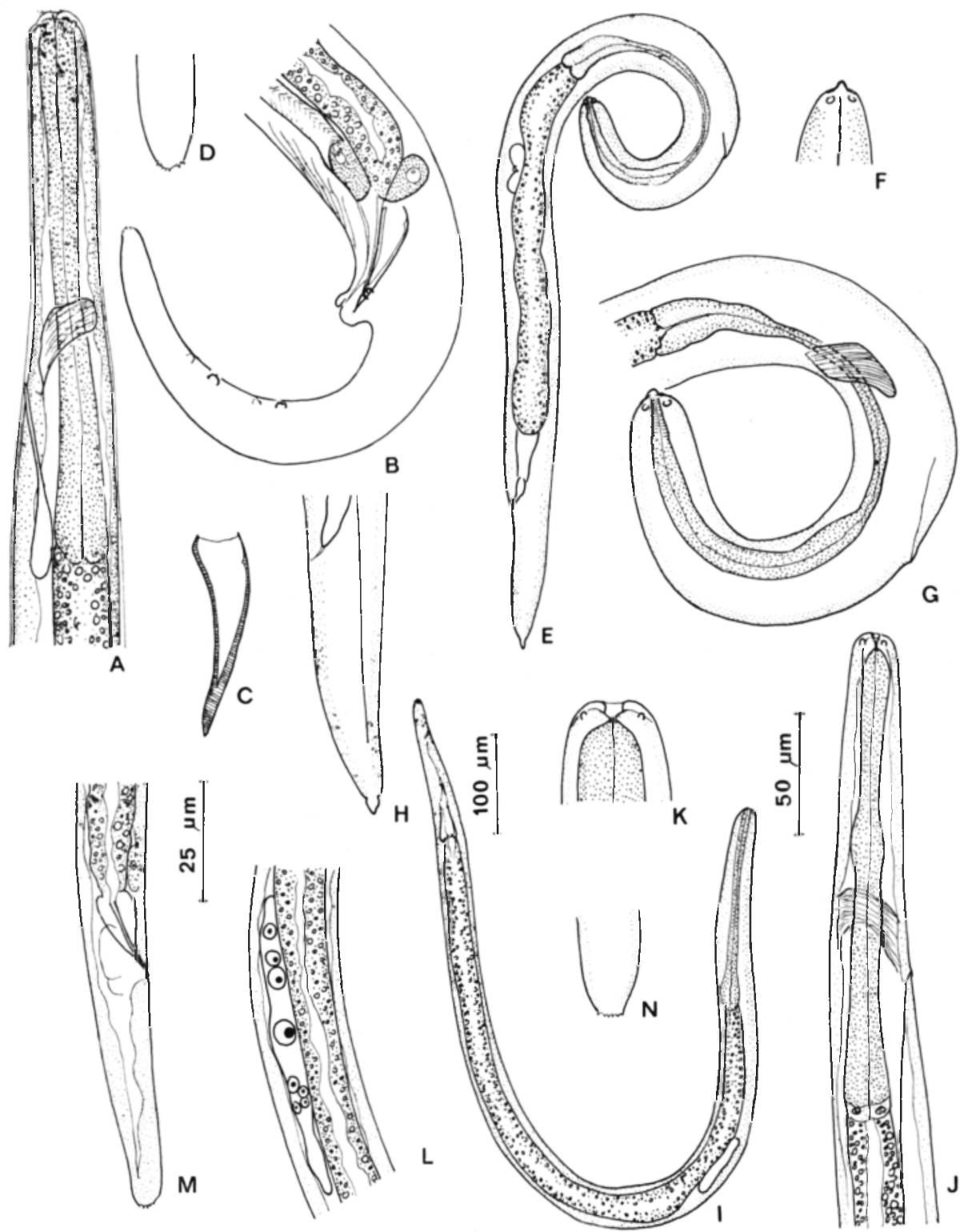

FIG. 1. - Skriabinelazia galliardi.

A-D : mâle : A, extrémité antérieure, vue latérale gauche ; $B$, extrémité postérjeure, vue latérale gauche; C, spicule; $D$, extrémité caudale.

$\mathrm{E}-\mathrm{H}$ : larve du $3^{\mathrm{e}}$ stade : $\mathrm{E}$, vue latérale gauche ; F, tête, vue latérale ; $\mathrm{G}$, cxtrémité céphalique, vue latérale gauche; $H$, queue, vue latérale gauche.

I-N : larve du 4 e stade : I, vue d'ensemble ; J, extrémité antérieure, vue latérale droite; $K$, tête vue latérale ; $L$, ébauche génitale, vue latérale droite; $M$, queue, vue latérale droile ; N, extrémilé caudale.

Echelles: A, B, E, G, .J, M, L : $50 \mu \mathrm{m} ; \mathrm{I}: 100 \mu \mathrm{m} ; \mathrm{C}, \mathrm{I}, \mathrm{F}, \mathrm{H}, \mathrm{K}, \mathrm{N}: 25 \mu \mathrm{m}$. 


\section{C - Larve femelle du Quatrik̀me stade}

Corps long de $1050 \mu \mathrm{m}$, large de $40 \mu \mathrm{m}$; petit plateau cuticulaire céphalique ; anneau nerveux et pore excréteur à 130 et $140 \mu \mathrm{m}$ de l'apex ; œsophage long de $200 \mu \mathrm{m}$; ébauche génitale en développement à $330 \mu \mathrm{m}$ de la tête ; queue longue de $95 \mu \mathrm{m}$ à extrémité arrondie et ornée de petites pointes.

\section{II - Données biologiques}

Le genre Skrjabinelazia Sypliaxova, 1930 (= Salobrella Teixeira de Freitas, 1940 ) est un genre particulièrement archaïque comme l'indiquent sa répartition géographique et ses particularités morphologiques.

Plusieurs publications (Sharpilo, 1976 ; Hasegawa, 1984; Roca et GarciaAdell, 1988) montrent la diversité des espèces et la très vaste répartition géographique du genre. Les neuf espèces signalées sont : S. taurica Sypliaxova, 1930, espèce-type, en Crimée; $S$. hoffmani I,i, 1934, en Chine ; S. intermedia (Freitas, 1940), au Brésil ; S. ornata Chabaud, Caballero et Brygoo, 1965, à Madagascar ; S. galliardi Chabaud, 1973, au Brésil ; S. machidai Hasegawa, 1984, au Japon; une nouvelle espèce (sous presse) de Roca et Garcia-Adell 1988, en Espagne, Skrjabinelazia sp. (Rizzo, 1902) (= Strongylus(?) n. sp. Rizzo, 1902) en Italie, et Skrjabinelazia sp. Angel et Mawson, 1960, en Australie. Toutes les neuf sont parasites de lézards insectivores.

La morphologie, très synthétique, fait transition entre celles de deux superfamilles elles-mêmes primitives : les Cosmocercoidea (Ascaridida) d'une part, et les Thelazioidea (Spirurida), d'autre part.

La biologie n'a pas encore été étudiée ; on sait seulement que toutes les espèces ont de très gros œufs à coque souple et fine contenant une larve complètement formée. Cependant $S$. galliardi présente plusieurs particularités.

Pahticularitís biologiques et morphologiques DE Skrjabinelazia galliardi

a) Il y a coexistence chez un même lézard de petits spécimens vivipares et de gros spécimens ovipares ; les spécimens vivipares n’ont qu'un très petit nombre de larves (une à trois) formées dans le tube génital ; un spécimen, de taille intermédiaire, a des œufs mùrs et plusieurs larves "écloses " dans l'utérus (Chabaud, 1973).

b) Les femelles ovipares renferment dans la moitié postérieure du corps des œufs très grands, à coque mince et souple, non colorée, contenant des larves mobiles et dans la moitié antérieure des œufs plus petits, à coque épaisse et rouge contenant des larves immobiles.

c) Les larves n'ont pas la petite râpe céphalique des larves du premier stade des Spirurida, ni le gros crochet cuticulaire dorsal de la larve du premier stade des Seuratum décrit par Quentin (1973). I a tête est armée d'une petite dent apicale 
renforcée à sa base par une couronne discontinue de très petits reliefs cuticulaires ; l'ensemble évoque plus la tête de la larve du troisième stade incluse dans l'ceuf d'un Hétérakide (cf. Strongyluris brevicauda étudié par Bain, 1970) qu'une larve du premier stade.

En outre, l'ébauche génitale multinucléée correspond aussi à une larve du troisième stade et non à celle d'un premier stade.

L'ensemble de ces constatations suggère donc la possibilité d'un cycle évolutif très aberrant.

\section{INFESTATION EXPÉRIMENTALE I'ARTHROPODES}

Des œufs non colorés, contenant des larves bien mobiles, et des œufs rouges, contenant des larves immobiles ont été placées dans l'eau, dans deux coupelles différentes; quelques éclosions ont été observées parmi les oufs clairs mais non parmi les ceufs rouges.

Quatre criquets, non identifiés, capturés à Belem, sont gavés individuellement avec le tiers antérieur des femelles ovipares, puis disséqués dans les jours qui suivent :

au quatrième jour $\mathrm{J}-4$ : un criquet avec une larve dans l'hèmocèle,

à $\mathrm{J}-7$ : un criquet négatif,

à $J-9$ : un criquet avec quatre larves dont la localisation n'a pas été précisée,

à $. J-14$ : un criquet avec quatre larves dans le tube digestif, deux dans l'ensemble tête-thorax et trois dans l'abdomen.

Toutes les larves récoltées ont une morphologie semblable à celle des larves contenues dans les œufs. Filles sont libres et mobiles, mais n'ont subi aucune évolution chez le criquet qui est donc un hòte paraténique.

\section{INTERPHÉTATION DES PARTIGULARITÉS CONSTATÉES}

Les résultats de l'infestation expérimentale confirment ainsi la morphologie : les larves contenues dans les ceufs sont au troisième stade larvaire. Il en est de même pour les larves trouvées libres dans l'utérus des femelles de petite taille, puisqu'elles ont toutes la même morphologie.

Le cycle évolutif doit donc s'interpréter de la façon suivante :

a) Les petites femelles larvipares paraissent biologiquement assimilables aux femelles d'Atractides : elles pondent des larves du $3^{\mathrm{e}}$ stade qui deviennent adultes directement dans l'intestin de l'hote et augmentent la population parasitaire lorsque la transmission à un nouvel individu hôte est difficile.

Dans le cas de Skrjabinelazia galliardi, et contrairement au cas des Atractides, la multiplication est très modérée ; seules quelques larves se trouvent dans l'utérus des femelles vivipares et le parasitisme des lézards reste faible.

b) La coexistence de petites femelles vivipares et de grosses femelles ovipares avait été interprétée implicitement comme un phénomène de poecilogynie tel qu'il est décrit chez les oxyures de lézards herbivores (Seurat, 1913; Petter, 1970). 
Un nouvel examen du matériel disponible indique au contraire qu'il n'y a pas deux sortes différentes de femelles, mais deux états physiologiques successifs. On constate en effet que les ovaires des femelles vivipares sont juvéniles, ils occupent les trois quarts du corps. Par ailleurs, un spécimen de taille intermédiaire a quelques larves libres et des oufs à coque épaisse. Nous pensons donc que ce sont les mêmes femelles qui sont vivipares lorsqu'elles sont juvéniles, puis ovipares lorsqu'elles sont mûres.

c) Ce ne sont pas les larves libres qui se trouvent incluses secondairement dans une coque ovulaire. Chez les femelles âgées, on observe dans la partie distale des utérus la présence de dizaines d'embryons se transformant en larves mobiles enfermées dans une coque mince et souple comme dans les autres espèces de Skrjabinelazia. Dans la partie proximale de l'utérus, ces larves sont progressivement incluses dans une coque épaisse et rigide chargée d'un pigment rouge et elles s'immobilisent. Il y a donc, comme chez les autres Nématodes la formation d'une coque ovulaire en trois couches, les deux premières, internes, formées par les enclaves de l'oocyte, la troisième, externe, sécrétée par la paroi utérine, mais, fait exceptionnel, celle-ci se constitue autour d'une larve du troisième stade.

\section{III - Conclusions}

Les grandes réussites évolutives dans les cycles biologiques des Nématodes parasites de vertébrés sont :

a) le développement des deux premiers stades larvaires dans le milieu extérieur : type Strongle;

b) une mue dans l'œuf, puis une pyramide d'hôtes de plus en plus spécifiques qui concentrent finalement le parasite chez son hôte définitif : type Ascaride,

c) le développement des deux premiers stades larvaires chez un invertébré hôte intermédiaire : type Spiruride,

d) deux mues dans l'œuf et l'ingestion directe par l'hôte définitif : type Oxyure.

Il y a eu des formes de passage entre ces différents grands types biologiques, mais elles n'ont pas donné lieu à de grandes explosions évolutives. Elles s'observent essentiellement dans des groupes archaïques faisant transition entre les grands ordres cités ci-dessus, ce qui est le cas précisément des Skrjabinelazia.

Dans les groupes voisins, nous connaissons :

les Cosmocercidés où les deux premières mues s'effectuent rapidement dans le milieu extérieur avec une croissance des larves faible ou nulle;

les Atractidés où le développement larvaire, s'effectuant entièrement dans l'intestin de l'hôte, il y a une multiplication intense du parasite qui permet le passage d'un hôte à l'autre par simple promiscuité et sans intervent ir n c ' 1 1 : 1: infestant spécialisé ; 
les Hétérakidés avec deux mues dans l'œuf et passage des stades infestants par un invertébré nécessaire ou facultatif ;

les Seuratidés, déjà proche du cycle hétéroxène des Spirurida, mais où le premier stade larvaire reste plusieurs jours dans l'intestin de l'arthropode sans évolution.

Les Skrjabinelazia vivent chez des lézards entomophages et ne peuvent ètre transmis que par l'intermédiaire d'invertébrés.

Plusieurs adaptations remarquables permettent au cycle de Skrjabinelazia galliardi de se fermer :

- les jeunes femelles vivipares assurent une reproduction de type Atractide qui semble limitée à quelques exemplaires, mais enrichit néanmoins le taux de parasitisme ;

- les femelles mûres pondent de nombreuses larves directement infestantes, mais protégées par une coque résistante;

-- les larves infestantes peuvent résister au moins 14 jours et vraisemblablement beaucoup plus dans le corps d'orthoptères et probablement d'autres invertébrés plus ou moins coprophages;

-- il est possible que la coque rouge vif des œufs soit un élément supplémentaire facilitant l'ingestion par des invertébrés.

En conclusion, Skrjabinelazia galliardi, espèce relique faisant transition entre les groupes de Nématodes importants et bien diversifiés que sont les Cosmocercoidea (Ascaridida) et les Thelazioidea (Spirurida) ont pu survivre pendant des millions d'années grâce à des adaptations multiples réalisant un cycle évolutif aberrant mais efficace.

Remerciemexts. - Le matériel a été récolté au cours d'une mission à l'Institut Evandro Chagas de Belen, grâce à l'obligeance du Dr. R. Lainson. Nous lui en exprimons notre reconnaissance.

\section{BIBLIOGRAPHIE}

Angel L. M., Mawson P. M. : Helminths from some lizards mostly from South Australia. Trans. Roy. Soc. S. Austr., 1968, 92, 59-72.

Bain O. : Cycle évolutif de l'Heterakidae Strongyluris brevicaudala (Nematoda). Mise en évidence de deux mues dans l'cuf. Ann. Parasitol. Hum. Comp., 1970, 45, 637-653.

Chanaud A. G. : Skrjabinelazia Sypliaxov, 1930 (= Salobrella Freitas, 1940), genre de transition entre Cosmocercidés et Spirurides. Ann. Parasitol. Hum. Comp., 1973, 48, 329-334.

Hasegawa H. : Skrjabinelazia machidai sp. n. (Nematoda: Seuratidae) from Gekko japonicus on Okinawa Island, Japan. Zool. Sci., 1984, 1, 483-486.

Petter A. J. : Deux cas de poecilogynie chez les Oxyures parasites d'Iguana iguana (I.). Bull. Mus. Nat. Hist. Nal., $2^{\mathrm{e}}$ sér., 1969 (1970), 41, 1252-1260.

Quentin J.-Cl. : Sur le cycle évolutif de Seuratum cadarachense Desportes, 1947 et ses affinités avec ceux des Nématodes Subulures (Ascaridida) et Rictulaires (Spirurida). Ann. Parasitol. Hum. Comp., 1970, 4i, 605-628.

Rizzo A. : La fauna elmintologica dei rettili. Arch. Parasitol. (Paris ), 1902, 6, 26-41.

Roca V., Garcia-Adell G. : Description de Skrjabinelazia pyreanica n. sp. (Nematoda, Seuratidae). Ann. Parasitol. Hum. Comp., 1988, 63, (sous presse).

Seurat L. G. : Sur un cas de poecilogynie chez un Oxyure. C. R. Soc. Biol., 1913, 74, 1089-1092.

Sharpilo V. P. : (Vers parasites des Reptiles d'URSSS). Izdatel'stvo "Naukova Dumka". Kiev, 220 p. (en russe, non consulté). 